\title{
The loudness dependence auditory evoked potential is insensitive to acute changes in serotonergic and noradrenergic neurotransmission
} \author{
K. Luan Phan ${ }^{6}$ and Pradeep J. Nathan ${ }^{1,4 *}$ \\ ${ }^{1}$ School of Psychology and Psychiatry, Monash University, Melbourne, VIC, Australia \\ ${ }^{2}$ Brain Sciences Institute, Swinburne University of Technology, Melbourne, VIC, Australia \\ ${ }^{3}$ School of Psychology, University of Wollongong, Wollongong, NSW, Australia \\ ${ }^{4}$ Brain Mapping Unit, Department of Psychiatry, University of Cambridge, Cambridge, UK \\ ${ }^{5}$ Clinical Unit Cambridge, GlaxoSmithKline Pharmaceuticals, Cambridge, UK \\ ${ }^{6}$ Department of Psychiatry, University of Michigan, Ann Arbor, Michigan, USA
}

Jessica Oliva ${ }^{1}$, Sumie Leung ${ }^{2}$, Rodney J. Croft ${ }^{3}$, Barry V. O’Neill ${ }^{4}$, Joanne O’Kane ${ }^{2}$, Julie Stout ${ }^{1}$,

\begin{abstract}
Background The loudness dependence of the auditory evoked potential (LDAEP) has been proposed as an electrophysiological marker for assessing serotonergic function in vivo in humans, although accumulating evidence suggests that it is insensitive to acute changes in serotonergic neurotransmission. Very little is known about the sensitivity of the LDAEP to other neurotransmitter systems including the noradrenergic system. The current study examined the effects of noradrenergic modulation as well as serotonergic modulation on the LDAEP. Methods The study utilised a double-blind placebo-controlled design in which the LDAEP in 17 healthy males and females was tested following acute administration of each of citalopram $(20 \mathrm{mg})$, reboxetine $(4 \mathrm{mg})$ and placebo.

Results Neither citalopram nor reboxetine modulated the LDAEP relative to placebo treatment $(p>0.05)$.

Conclusion These findings suggest that the LDAEP is insensitive to acute changes in serotonergic or noradrenergic neurotransmission and thus is a poor pharmacodynamic marker of these systems. Copyright (C) 2010 John Wiley \& Sons, Ltd.
\end{abstract}

KEY WORDS - LDAEP; serotonin; noradrenaline; biological marker; electrophysiology; healthy participants

\section{INTRODUCTION}

The loudness dependence of auditory evoked potentials (LDAEPs) has been proposed as a non-invasive biological marker of in vivo central serotonergic function (Hegerl and Juckel, 1993). It is a measure of auditory cortex activity, reflecting an increase or decrease in the slope of auditory evoked potentials with increasing tone loudness (Hegerl and Juckel, 1993). It has been postulated that the excitability of the auditory cortex is under serotonergic control, such that an increase or decrease in serotonin function is associated with reduced or increased cortical excitability (e.g. decrease and increase in the slope of the LDAEP, respectively).

\footnotetext{
* Correspondence to: P. J. Nathan, Brain Mapping Unit, Department of Psychiatry, University of Cambridge, Addenbrooke's Hospital, Hills Road, Cambridge CB2 2QQ, UK. Tel: +44 (0) 1223 296000, Fax: +44 (0) 1223 296002. E-mail: pn254@cam.ac.uk
}

The majority of evidence in support of an association between serotonin function and the LDAEP has been based on pharmacological studies in animals (Juckel et al., 1997, 1999), with additional indirect evidence from clinical disorders with a presumed serotonergic dysfunction including migraine (Wang et al., 1999), borderline personality disorder (Norra et al., 2003), generalised anxiety disorder (Senkowski et al., 2003) and schizophrenia (Juckel et al., 2003). However, human studies that have directly examined the effects of serotonergic modulation on the LDAEP have been unable to support the animal studies (Juckel et al., 1997, 1999). In one human study, we showed that the slope of the LDAEP was reduced by acutely enhancing synaptic serotonin with the selective serotonin reuptake inhibitor (SSRI) citalopram (Nathan et al., 2006). However, subsequent studies, including our own, have shown insensitivity of the LDAEP to enhancement of serotonin neurotransmission following acute administration of the SSRI's citalopram (Guille 
et al., 2008; Uhl et al., 2006), escitalopram or sertraline (Guille et al., 2008). Similarly, depletion of serotonin with depletion of the serotonin precursor, tryptophan was also found to have no effect on the LDAEP in the majority of studies (Dierks et al., 1999; Norra et al., 2008; O'Neill et al., 2008a) while one study reported a paradoxical decrease in the LDAEP (Kähkönen et al., 2002). Overall these findings suggest that the LDAEP may be insensitive to acute changes in serotonin neurotransmission (O'Neill et al., 2008b).

Relatively little is known about the impact of other neurotransmitter systems on the LDAEP. A greater understanding of the sensitivity of the LDAEP to multiple neurotransmitter systems is required because of the reported changes in the LDAEP in clinical disorders (Juckel et al., 2003; Norra et al., 2003; Senkowski et al., 2003; Wang et al., 1999) and the lack of knowledge about the underlying influence of neurochemicals other than serotonin. We have recently shown that the LDAEP is sensitive to NMDA receptor modulation (O'Neill et al., 2007) but insensitive to dopamine $\mathrm{D}_{1}$ and $\mathrm{D}_{2}$ receptor stimulation (O'Neill et al., 2006) and dopamine depletion (O'Neill et al., 2008a). It is yet to be established whether the LDAEP is directly modulated by changes in noradrenergic neurotransmission in healthy subjects, although it has been recently demonstrated that the LDAEP at baseline may be a predictor of treatment response to noradrenergic antidepressants in a clinical sample of patients with depression (Juckel et al., 2007; Linka et al., 2009). Hence, the current study examined the acute effects of noradrenergic modulation using the noradrenaline reuptake inhibitor, reboxetine, on the LDAEP in healthy subjects. Due to inconsistent reports with SSRIs, we also further examined the effects of the SSRI citalopram, on the LDAEP.

\section{METHODS}

\section{Participants}

The sample consisted of 21 healthy, drug naive, participants aged 18-32 (mean age 22.05, SD 3.44) with no current or past history of psychiatric or neurological disorders confirmed by a semi-structured medical examination by a registered physician. All participants provided written informed consent to take part in the study, which was approved by both the Swinburne and Monash University Human Research Ethics Committees.

\section{Design}

The study utilised a double-blind, placebo-controlled within-subject design. Participants were tested under three acute treatment conditions: citalopram (Cipramil, $20 \mathrm{mg}$ ), reboxetine (Edronax, $4 \mathrm{mg}$ ) and placebo. Order of assignment to treatment groups was randomised and counterbalanced, separated by a minimum 7-day washout period. Females were tested during the follicular phase of their menstrual cycle.

\section{Procedure}

The study was conducted at the Brain Sciences Institute. On the day of testing, participants were administered citalopram, reboxetine or placebo at approximately $10 \mathrm{am}$. Event-related potential recordings were performed $2 \mathrm{~h}$ post-treatment to coincide with peak pharmacokinetic effects of both reboxetine $(2 \mathrm{~h}$; Dostert et al., 1997) and citalopram (2-4 h; Hyttel, 1994) and pharmacodynamic effects of citalopram (Kemp et al., 2004).

\section{Data acquisition}

Electroencephalographic (EEG) data were recorded as described in our previous studies (Nathan et al., 2006; O'Neill et al., 2006, 2007). This included EEG recording using tin electrodes from 61 scalp sites according to the international $10 / 20$ system using a Quickcap as well as $9 \mathrm{~mm}$ diameter tin electrodes attached at the mastoids for re-referencing, and below the left eye for recording eye movement. Auditory stimuli of the loudness dependency paradigm consisted of fifty $1000 \mathrm{~Hz}$ tones $(1000 \mathrm{~Hz}, 100 \mathrm{~ms}$ duration including $10 \mathrm{~ms}$ rise and $10 \mathrm{~ms}$ fall time, SOA randomised between 1600 and $2100 \mathrm{~ms}$ ) at a range of intensities $(60,70,80,90$ and $100 \mathrm{~dB})$, presented binaurally in a pseudorandom order through single-use foam EAR inserts. Participants were also presented with a series of faces and asked to respond with a button press if the face had a nose. The purpose of this visual task was to distract attention away from the auditory stimuli as attention has been shown to modulate the LDAEP in humans (Baribeau and Laurent, 1987). Data were collected with a sampling rate of $500 \mathrm{~Hz}$ and a bandpass filter of $0.5-100 \mathrm{~Hz}$. The data were recorded using NeuroScan software with SynAmps2 $2^{\mathrm{TM}}$ amplifiers (NeuroScan).

\section{Data analysis}

Electroencephalogram data were digitally re-referenced to the average recorded from the mastoid electrodes, low-pass filtered at $30 \mathrm{~Hz}(24 \mathrm{~dB} / \mathrm{oct})$ and epoched $-100-400 \mathrm{~ms}$ post-auditory stimulus. Ocular artefact was corrected using the Croft and Barry (2000) 
method, whereby epochs with data exceeding $\pm 75 \mu \mathrm{V}$ were rejected and averages then created for each of the five intensities separately. Averages were created for each of the five intensities separately with a mean of $47.8 \pm 2.2$ sweeps per intensity. On the grand mean waveform, the $\mathrm{N} 1$ and $\mathrm{P} 2$ peaks were identified at 100 and $185 \mathrm{~ms}$ respectively at the $\mathrm{Cz}$ electrode. The N1 and $\mathrm{P} 2$ peaks were then detected from the session mean waveforms of each participant at 70-130 and 110 $260 \mathrm{~ms}$ respectively. The latencies of the session mean peaks were then used to determine the peak detection time ranges for each intensity waveform of that session (N1: $\pm 15 \mathrm{~ms} ; \mathrm{P} 2: \pm 30 \mathrm{~ms}$ ). The peaks were determined automatically at the $\mathrm{Cz}$ electrode and subsequently visually inspected by the investigator to ensure accuracy. N1/P2 amplitude was calculated as the differences in amplitude between P2 and N1. The slope of the N1/P2 was calculated as the least squares linear regression slope, with stimulus intensity the independent variable and N1/P2 amplitude the dependent variable.

\section{Statistical analysis}

A repeated measures linear contrast was performed to determine whether N1/P2 amplitude increased linearly with stimulus intensity in the placebo condition, and two repeated measures planned contrasts were performed to test for an effect of citalopram and reboxetine (compared to placebo) on the slope of the LDAEP. Gender was employed as a between-subjects factor to reduce error variance as gender has been found to influence visual amplitude-intensity slopes (Buchsbaum and Pfefferbaum, 1971; Camposano and Lolas, 1992). Because these two comparisons were based on a theoretically driven rationale and because there are fewer tests than degrees of freedom, each planned contrast had an alpha level set at 0.05 (Tabachnick and Fidell, 2007).

\section{RESULTS}

Four people were excluded from the analysis because recognisable ERP components (and in particular the N1/P2 complex) could not be discerned in the waveforms. The remaining samples $[n=17(M=8, F=9)]$ are presented here. The N1/P2 amplitude increased linearly with increasing stimulus intensity in the placebo condition $\left(F_{(1,16)}=42.67, p<0.0001\right.$, partial $\eta^{2}=0.73$ ) (Figure 1).

The mean slopes for placebo, citalopram and reboxetine were $0.33,0.38$ and 0.30 respectively (Figure 2).

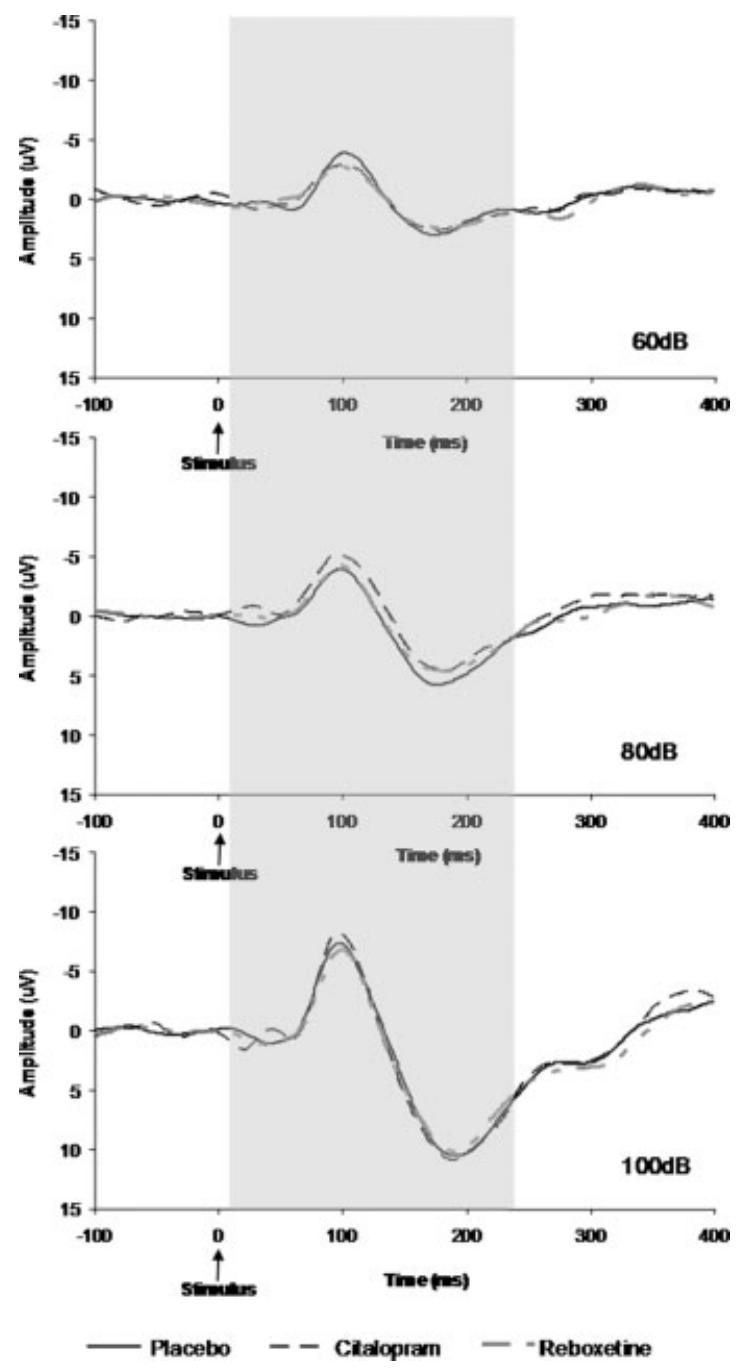

Figure 1. Grand mean auditory evoked potentials at $\mathrm{Cz}$ for placebo, citalopram and reboxetine treatment conditions at 60, 80 and $100 \mathrm{~dB}$ intensities of the auditory stimuli

Compared to placebo, there was no significant effect of citalopram $\left(F_{(1,15)}=0.09, p>0.05\right.$, partial $\left.\eta^{2}=0.006\right)$ or reboxetine $\left(F_{(1,15)}=3.92, p>0.05\right.$, partial $\eta^{2}=$ 0.207 ) on the LDAEP slope (Figure 2).

\section{DISCUSSION}

Acutely enhancing serotonergic or noradrenergic neurotransmission with citalopram and reboxetine respectively did not modulate the LDAEP. These findings are consistent with the majority of studies conducted in humans that have similarly shown that acute enhancement of synaptic serotonin with the SSRI's citalopram (Guille et al., 2008; Uhl et al., 2006), escitalopram and sertraline (Guille et al., 2008) and depletion of synaptic 

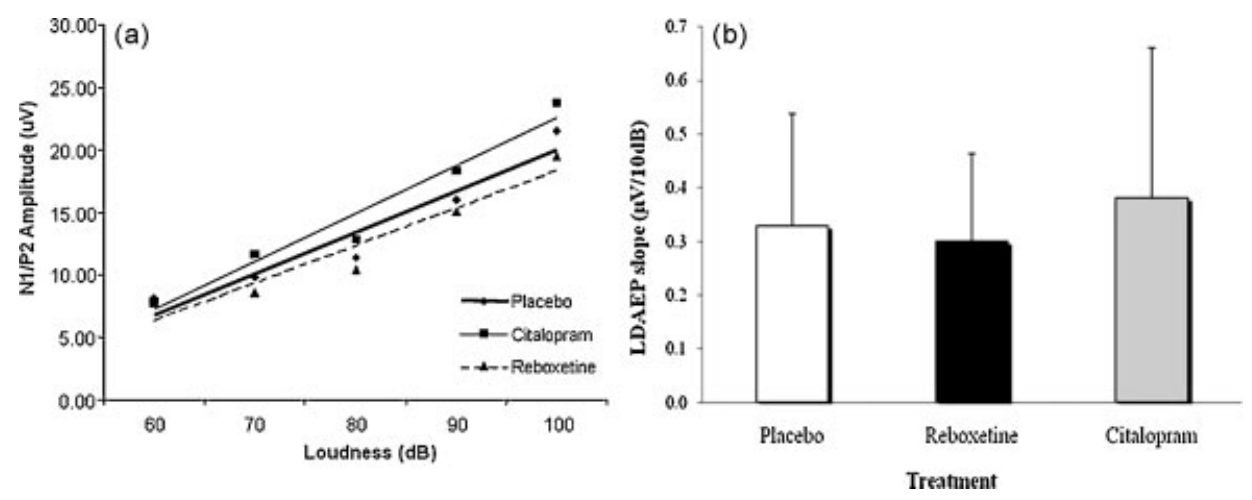

Figure 2. (a) Mean N1/P2 amplitude ( $\mu \mathrm{V}$ ) plotted against stimulus intensity (loudness, $\mathrm{dB}$ ) and (b) mean values (mean \pm standard deviation) of LDAEP $(\mu \mathrm{V} / 10 \mathrm{~dB})$ at $\mathrm{Cz}$, for placebo, citalopram and reboxetine treatment conditions

serotonin using tryptophan depletion (O'Neill et al., 2008a) has no effect on the LDAEP. The insensitivity of the LDAEP to noradrenergic modulation supports previous studies in animals and humans. For example, the $\alpha_{2}$-receptor agonist clonidine was found to have had no effect on the LDAEP in cats (Juckel et al., 1997), and in humans, a lack of association was reported between the noradrenaline metabolite, 3-methoxy-4-hydroxyphenylglycol (MHPG), in CSF and the amplitude-intensity function of visual evoked potentials (Von Knorring and Perris, 1981).

Based on these studies it could be argued that the LDAEP is insensitive to acute changes in serotonergic or noradrenergic neurotransmission and hence the LDAEP may be considered a poor pharmacodynamic marker of these neurochemical systems. Although it is possible that the LDAEP may be sensitive to long-term changes in serotonergic function (for a review see O'Neill et al., 2008b) and thus may potentially be a trait or biological marker, this is yet to be directly demonstrated. Together, the findings suggest that the changes in the LDAEP previously reported in clinical disorders (Juckel et al., 2003; Norra et al., 2003; Senkowski et al., 2003; Wang et al., 1999) may not be entirely attributed to a dysfunction in the serotonergic system as there is insufficient consistent data linking changes in serotonergic neurotransmission and the LDAEP and such a relationship is yet to be directly shown following long-term modulation of the serotonin neurotransmission. As we have previously shown that the LDAEP is sensitive to NMDA receptor modulation (O'Neill et al., 2007), it is possible that the LDAEP changes observed in clinical disorders including schizophrenia and mood disorders may be secondary to glutamatergic dysfunction (Laruelle et al., 2003; Sanacora et al., 2008). Future studies focussing on the effects of long-term modulation of the serotoni- nergic (and other systems) on the LDAEP will help clarify the neurochemical basis of the LDAEP changes observed in clinical disorders.

There are some methodological issues that warrant discussion. First, the negative findings are unlikely due to inadequate power as a statistical analysis showed that the effect sizes were small and greater than 200 subjects would be required to achieve significant effects (with a power of 0.8 at alpha 0.05 ). Second, this study used scalp data to determine the slope of the LDAEP. Previous studies have claimed that the dipole source localisation method is more sensitive than the scalp-derived method (Hegerl et al., 1994; Hegerl and Juckel, 1993) as it can separate the tangential dipole which may be more sensitive to changes in serotonin neurotransmission (Campbell et al., 1987). However, as yet, the advantage of dipole source localisation method over the scalp-derived method has not been directly demonstrated. Furthermore, we recently showed that the dipole source analysis is no more sensitive than the scalp-derived method in detecting the acute effects of citalopram on the LDAEP (Guille et al., 2008). As such, it is unlikely that the negative findings in this study are a result of the data being analysed using the scalp-derived method.

In summary, acute enhancement of serotonergic or noradrenergic neurotransmission using citalopram and reboxetine respectively had no effects on the LDAEP. These findings together with previous findings in the literature suggest that the LDAEP is a poor pharmacodynamic marker of the serotonin and noradrenergic systems.

\section{ACKNOWLEDGEMENTS}

PJN and BVO are employees of GlaxoSmithKline Pharmaceuticals. 


\section{REFERENCES}

Baribeau JC, Laurent JP. 1987. The effect of selective attention on augmenting/intensity function of the early negative waves of AEPs. Electroencephalogr Clin Neurophysiol Suppl 40: 68-75.

Buchsbaum M, Pfefferbaum A. 1971. Individual differences in stimulus intensity response. Psychophysiology 8: 600-611.

Campbell MJ, Lewis DA, Foote SL, Morrison JH. 1987. Distribution of choline acetyltransferase-, serotonin-, dopamine-beta-hydroxylase-, tyrosine hydroxylase-immunoreactive fibers in monkey primary auditory cortex. J Comp Neurol 261: 209-220.

Camposano S, Lolas F. 1992. Effects of stimulation intensity, gender and handedness upon auditory evoked potentials. Arq Neuropsiquiatr 50: $43-$ 49.

Croft RJ, Barry RJ. 2000. EOG correction of blinks with saccade coefficients: a test and revision of the aligned artefact average solution. Clin Neurophysiol 111: 444-451.

Dierks T, Barta S, Demisch L, et al. 1999. Intensity dependence of auditory evoked potentials (AEPs) as biological marker for cerebral serotonin levels: effects of tryptophan depletion in healthy subjects. Psychopharmacology 146: 101-107.

Dostert P, Benedetti MS, Poggesi I. 1997. Review of the pharmacokinetics and metabolism of reboxetine, a selective noradrenaline reuptake inhibitor. Eur Neuropsychopharmacol 7: S23-S35.

Guille V, Croft RJ, O’Neill BV, Illic S, Phan KL, Nathan PJ. 2008. An examination of acute changes in serotonergic neurotransmission using the loudness dependence measure of auditory cortex evoked activity: effects of citalopram, escitalopram and sertraline. Hum Psychopharmacol 23: 231-241.

Hegerl U, Gallinat J, Mrowinski D. 1994. Intensity dependence of auditory evoked dipole source activity. Int J Psychophysiol 17: 1-13.

Hegerl U, Juckel G. 1993. Intensity dependence of auditory evoked potentials as an indicator of central serotonergic neurotransmission: a new hypothesis. Biol Psychiatry 33(3): 173-187.

Hyttel J. 1994. Pharmacological characterization of selective serotonin reuptake inhibitors (SSRIs). Int Clin Psychopharmacol 9(Suppl 1): $19-26$.

Juckel G, Gallinat J, Riedel M, et al. 2003. Serotonergic dysfunction in schizophrenia assessed by the loudness dependence measure of primary auditory cortex evoked activity. Schizophr Res 64: 115-124.

Juckel G, Hegerl U, Molnar M, Csepe V, Karmos G. 1999. Auditory evoked potentials reflect serotonergic neuronal activity - a study in behaving cats administered drugs acting on 5-HT1A autoreceptors acting in the dorsal raphe nucleus. Neuropsychopharmacology 21: 710-716.

Juckel G, Molnar M, Hegerl U, Csepe V, Karmos G. 1997. Auditory evoked potentials as indicator of brain serotonergic activity - first evidence in behaving cats. Biol Psychiatry 41: 1181-1195.

Juckel G, Pogarell O, Augustin H, et al. 2007. Differential prediction of first clinical response to serotonergic and noradrenergic antidepressants using the loudness dependence of auditory evoked potentials in patients with major depressive disorder. J Clil Psychiatr 68: 1206-1212.

Kähkönen S, Jääskeläinen IP, Pennanen S, Liesivuori J, Ahveninen J. 2002. Acute tryptophan depletion decreases intensity dependence of auditory evoked magnetic N1/P2 dipole source activity. Psychopharmacology 164: 221-227.
Kemp AH, Gray MA, Silberstein RB, Armstrong SM, Nathan PJ. 2004. Augmentation of serotonin enhances pleasant and suppresses unpleasant cortical electrophysiological responses to visual emotional stimuli in humans. Neuroimage 22(3): 1084-1096.

Laruelle M, Kegeles LS, Abi-Dargham A. 2003. Glutamate, dopamine, and schizophrenia: from pathophysiology to treatment. Ann NY Acad Sci 1003: $138-158$.

Linka T, Sartory G, Wiltfang J, Muller BW. 2009. Treatment effects of serotonergic and noradrenergic antidepressants on the intensity dependence of auditory ERP components in major depression. Neurosci Lett 463: $26-30$.

Nathan PJ, Segrave R, Phan KL, O’Neill B, Croft RJ. 2006. Direct evidence that acutely enhancing serotonin with the selective serotonin reuptake inhibitor citalopram modulates the loudness dependence of the auditory evoked potential (LDAEP) marker of central serotonin function. Hum Psychopharmacol 21(1): 47-52.

Norra C, Becker S, Brocheler A, Kawohl W, Kunert HJ, Buchner H. 2008. Loudness dependence of evoked dipole source activity during acute serotonin challenge in females. Hum Psychopharmacol 23: 31-42.

Norra C, Mrazek M, Tuchtenhagen F, et al. 2003. Enhanced intensity dependence as a marker of low serotonergic neurotransmission in borderline personality disorder. J Psychiatr Res 37: 23-33.

O’Neill BV, Croft RJ, Leung S, et al. 2006. Dopamine receptor stimulation does not modulate the loudness dependence of the auditory evoked potential in humans. Psychopharmacology 188(1): 92-99.

O’Neill BV, Croft RJ, Leung S, Oliver C, Phan KL, Nathan PJ. 2007. Highdose glycine inhibits the loudness dependence of the auditory evoked potential (LDAEP) in healthy humans. Psychopharmacology (Berl) 195(1): 85-93.

O'Neill BV, Guille V, Croft RJ, et al. 2008a. Effects of selective and combined serotonin and dopamine depletion on the loudness dependence of the auditory evoked potential (LDAEP) in humans. Hum Psychopharmacol 23: 301-312.

O’Neill BV, Croft RJ, Nathan PJ. 2008b. The loudness dependence of the auditory evoked potential (LDAEP) as an in vivo biomarker of central serotonergic function in humans: rationale, evaluation and review of findings. Hum Psychopharmacol 23: 355-370.

Sanacora G, Zarate CA, Krystal JH, Manji HK. 2008. Targeting the glutamatergic system to develop novel, improved therapeutics for mood disorders. Nat Rev Drug Discov 7(5): 426-437.

Senkowski D, Linden M, Zubragel D, Bar T, Gallinat J. 2003. Evidence for disturbed cortical signal processing and altered serotonergic neurotransmission in generalized anxiety disorder. Biol Psychiatry 53: 304314.

Tabachnick B, Fidell L. 2007. Using Multivariate Statistics. Pearson Education: New Jersey.

Uhl I, Gorynia I, Gallinat J, et al. 2006. Is the loudness dependence of auditory evoked potentials modulated by the selective serotonin reuptake inhibitor citalopram in healthy subjects? Hum Psychopharmacol 21(7): 463-471.

Von Knorring L, Perris C. 1981. Biochemistry of the augmenting/reducing response in visual evoked potentials. Neuropsychobiology 7: 1-8.

Wang W, Wang YH, Fu XM, Sun ZM, Schoenen J. 1999. Auditory evoked potentials and multiple personality measures in migraine and post-traumatic headaches. Pain 79: 235-242. 